\title{
Detection of Disulfides in Protein Extracts of Arabidopsis thaliana Using Monobromobimane (mBB) \\ Shin-nosuke Hashida ${ }^{1, *}$ and Maki Kawai-Yamada²
}

\begin{abstract}
1Environmental Science Research Laboratory, Central Research Institute of Electric Power Industry, Abiko, Japan; ${ }^{2}$ Graduate School of Science and Engineering, Saitama University, Saitama, Japan *For correspondence: shashida@criepi.denken.or.jp
\end{abstract}

\begin{abstract}
[Abstract] Thiol-disulfide exchange is a key posttranslational modification, determining the folding process of intra- and inter-protein structures. Thiols can be detected by colorimetric reagents, which are stoichiometrically reduced by free thiols, and by fluorescent adducts, showing fluorescence only after thioester formation. We adapted a simple three-step method for detection of disulfide bonds in proteins. After irreversible blocking of protein thiols, disulfide bonds are reduced, followed by the detection of thiols. The approach presented here provides an economical procedure that can be used to obtain a global overview of the thiol-disulfide status of proteins in plants. This method allows the detection of modifications in samples on a gel and can be used for semi-quantitative analysis.
\end{abstract}

Keywords: Protein labeling, Thiol, Disulfide, Monobromobimane (mBB), Tris(2-carboxyethyl) phosphine (TCEP), Redox, Arabidopsis thaliana

[Background] The redox status of proteins plays a key role in a number of cellular processes. Thioldisulfide exchange reactions support the redox control of plant responses to fluctuating light under natural environments via a photochemical electron transfer cascade for posttranslational modification of proteins. Whereas thiols (R-SH) have relatively higher reactivity than other cellular components and can be directly detected by a variety of reagents and separation techniques (Fahey et al., 1980 and 1981; Newton et al., 1981; Riddles et al., 1983; Fenton and Fahey, 1986; Tyagarajan et al., 2003), disulfide bonds (R-S-S-R) have no such chemical characteristics. Therefore, the most common methodology for the detection of disulfide bonds consists of a three-step procedure: blocking of thiols, reduction of disulfide bonds, and subsequent detection of additionally exposed thiols. Dithiothreitol (DTT) is a wellknown and frequently used reducing agent for the protection and exposure of thiol groups of proteins. However, the use of DTT in the laboratory is undesirable because of its harmful odor. Moreover, it can subsequently interfere with thiol detection reagents unless it is appropriately removed (Getz et al., 1999). Also, the reducing power of DTT is limited to $\mathrm{pH}>7$. Tris(2-carboxyethyl) phosphine-hydrochloride (TCEP) can be a promising alternative with advantages of being odorless and more effective in the reduction of disulfide bonds over a wide $\mathrm{pH}$ range from 1.5 to 8.5. Monobromobimane (mBB) has been used for fluorescent labeling of low molecular weight thiols by detecting fluorescent derivatives under a fluorescent microscope or with high-performance liquid chromatography (Fahey and Newton, 1987; Meyer et al., 2001). This protocol describes a simple method for discriminate labeling with mBB of free thiols, disulfide bonds, and total thiols in plant protein extracts. Here, we show an example of the 
procedure using Arabidopsis leaves. Free thiols are labeled without any preprocessing (Figure 1A), whereas labeling of disulfide bonds requires blocking of free thiols by iodoacetamide (IAA) and reduction of disulfide bonds by TCEP before the mBB treatment (Figure 1B). Total labeling of thiols just after TCEP treatment is helpful to estimate the redox state of total thiols in protein extracts (Figure 1C). This simple method allows the detection of modifications in samples on a gel and can be used for semi-quantitative analysis and provides an overview of the redox state of thiol-containing proteins without any special equipment other than conventional protein electrophoresis and imaging systems.

A

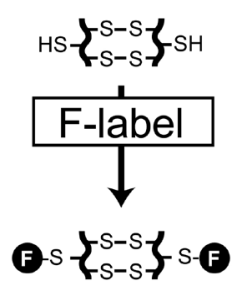

B

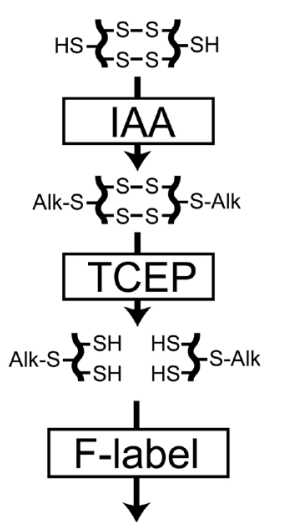

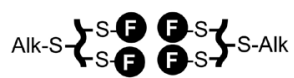

C

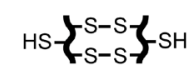

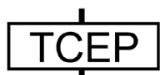

HS $\mathcal{F}_{-S H}^{-S H}$ HS $\}_{S H}$

I

F-label

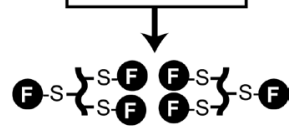
(l)

Figure 1. Flowchart for discriminate labeling of thiols in protein under different redox states.

A. Labeling of free thiols in proteins. B. Specific labeling of thiols, forming disulfide bonds in protein.

$C$. Total labeling of thiols in proteins. $F$ in closed circle means $m B B-d e r i v e d$ fluorescence. IAA: lodoacetamide, TCEP: Tris(2-carboxyethyl)phosphine Hydrochloride.

\section{Materials and Reagents}

1. Finntip Pipette Tips (Finntip Flex 10, 200, and 1,000)

2. $1.5 \mathrm{ml}$ microcentrifuge tube (VIOLAMO, F-1.5, catalog number: 1-1600-01)

3. Arabidopsis thaliana wild-type plants (Ecotype: Col-0)

4. Arabidopsis thaliana mutant plant of interest (Ecotype: Col-0)

5. Liquid nitrogen

6. Tris(hydroxymethyl)aminomethane (Tris) (Nacalai Tesque, catalog number: GR35406-91)

7. cOmplete ${ }^{T M}$, Mini, Protease Inhibitor Cocktail (Roche, catalog number: 4693159001)

8. Bio-Rad Protein Assay Kit (Bio-Rad, catalog number: 5000001ja)

9. FOCUS ${ }^{\mathrm{TM}}$ Protein Alkylation (TaKaRa, catalog number: GA503)

10. lodoacetamide (IAA) (Sigma-Aldrich, catalog number: I1149-5G)

11. Trichloroacetic acid solution (TCA), $100 \%$ (w/v) (Nacalai Tesque, catalog number: SP06275-24)

12. Acetone (Nacalai Tesque, catalog number: SP09888-85)

13. FOCUS ${ }^{\mathrm{TM}}$ Protein Reductant (TaKaRa, catalog number: GA501) 
14. Tris(2-carboxyethyl)phosphine Hydrochloride (TCEP) (Nacalai Tesque, catalog number: SP06342-21)

15. Monobromobimane (mBB) (Tokyo Chemical Industry, catalog number: B4220)

16. Glycerol (Nacalai Tesque, catalog number: SP17045-94)

17. Bromophenol Blue (BPB) (Nacalai Tesque, catalog number: B-2609)

18. Glycine (Nacalai Tesque, catalog number: SP17141-24)

19. Sodium lauryl sulfate (SDS) (Nacalai Tesque, catalog number: SP08933-34)

20. Protein extraction buffer (see Recipes)

21. IAA solution (see Recipes)

22. SDS-PAGE sample buffer (see Recipes)

23. SDS-PAGE buffer (see Recipes)

\section{Equipment}

1. Pipettes (Thermo Fisher Scientific, Finnpipette ${ }^{T M} F 2$ )

2. Cork borer ( $4.0 \mathrm{~mm}$, inside diameter of the hole)

3. Conventional Dewar's vessel

4. Vortexer (Scientific Industries, Vortex-Genie 2)

5. Homogenizer pestle (VIOLAMO, F-1.5, catalog number:1-2955-02)

6. Refrigerated microcentrifuge (TOMY, MX305)

7. Absorption spectrometer (Thermo Fisher Scientific, Multiskan ${ }^{\mathrm{TM}} \mathrm{GO}$ )

8. Mini-PROTEAN Tetra System (Bio-Rad, catalog number: 1658004ja)

9. Mini-PROTEAN TGX Gels (Bio-Rad, catalog number: 456-1095)

10. Power supply (ATTO, catalog number: AE-8135)

11. UV Transilluminator (Maestrogen, model: UV-01)

12. Yellow filter (Kenko, Black \& White Filter, Y2 Professional, $\lambda_{\text {cut-on }} 470-480 \mathrm{~nm}$ )

13. Conventional digital camera with hood (RICHO, WG-4)

\section{Software}

1. ImageJ (https://imagej.nih.gov/ij//)

\section{Procedure}

1. Protein extraction

a. Place freshly prepared leaf discs $(10-50 \mathrm{mg})$ punched-out with a cork borer in a $1.5 \mathrm{ml}$ microcentrifuge tube and immediately freeze in liquid nitrogen in a Dewar's vessel. The estimated average weight of five leaf discs should be approximately $10 \mathrm{mg}$.

b. Grind the sample in the microcentrifuge tube with a homogenizer pestle and add $200 \mu \mathrm{l}$ of 
the ice-cold protein extraction buffer (prepare immediately prior to use, Recipe 1) per $50 \mathrm{mg}$ sample on ice before sample lysis.

Note: Make sure not to dissolve the sample. We recommend grinding the sample in the presence of liquid nitrogen rather than on ice. In the case of Arabidopsis leaf discs, it is easy to obtain fully homogenized sample in a couple of seconds.

c. Centrifuge at $14,000 \times \mathrm{g}, 4^{\circ} \mathrm{C}$ for $10 \mathrm{~min}$.

d. Transfer the supernatant to a new $1.5 \mathrm{ml}$ microcentrifuge tube for removing sample debris.

2. Protein measurement

a. Prepare the dye for Bio-Rad Protein Assay by diluting 1 volume of the dye reagent concentrate with 4 volumes of distilled, deionized (DDI) water.

b. Add 2 and $5 \mu$ protein extract into 198 and $195 \mu$ diluted dye reagent, respectively.

c. After $5 \mathrm{~min}$, measure absorbance at $595 \mathrm{~nm}$ and estimate the protein concentration with protein standard II (BSA) in Bio-Rad Protein Assay Kit (0.2 to $2.0 \mathrm{mg} / \mathrm{ml})$.

3. Thiol alkylation

a. Adjust the protein concentration to $1.0 \mathrm{mg} / \mathrm{ml}$ with the extraction buffer and combine several samples to prepare $1.5 \mathrm{ml}$ of protein extract in a single tube.

b. Divide the solution into three $1.5 \mathrm{ml}$ microcentrifuge tubes. Each tube contains $500 \mu \mathrm{l}$ of the extract, one each for the determination of free thiols (tube A), disulfide bonds (tube B), and total thiols (tube $\mathrm{C}$ ) in the protein extracts. All the tubes should be kept on ice until use.

Note: For all experiments, we strongly recommend starting from a single protein extract to discriminately detect free thiols, disulfide bonds, and total thiols in proteins to minimize the sample variations.

c. Add $2.5 \mu \mathrm{l} \mathrm{FOCUS}{ }^{\mathrm{TM}}$ alkylation buffer to tubes $\mathrm{B}$ and $\mathrm{C}$, and vortex for $10 \mathrm{~s}$.

d. Add $25 \mu \mathrm{l} \mathrm{0.4} \mathrm{M} \mathrm{IAA} \mathrm{(prepare} \mathrm{immediately} \mathrm{prior} \mathrm{to} \mathrm{use,} \mathrm{Recipe} \mathrm{2)} \mathrm{only} \mathrm{in} \mathrm{tube} \mathrm{B} \mathrm{for} \mathrm{detection}$ of disulfide bonds.

e. Incubate tubes $\mathrm{B}$ and $\mathrm{C}$ without shaking at room temperature (approximately $23^{\circ} \mathrm{C}$ ), under dark for $30 \mathrm{~min}$.

4. Protein purification

a. Add an equal volume of cold $20 \%(\mathrm{w} / \mathrm{v})$ TCA solution to tubes $B$ and $\mathrm{C}$.

b. Centrifuge the tubes at $14,000 \times \mathrm{g}, 4^{\circ} \mathrm{C}$ for $30 \mathrm{~min}$.

c. Remove the supernatant, leaving the protein pellet intact.

d. Remove TCA by washing pellet twice with $500 \mu \mathrm{l}$ cold acetone and retain the pellet.

5. Disulfide reduction

a. Adjust the protein reduction solution by adding $2.5 \mu \mathrm{l} \mathrm{FOCUS}{ }^{\mathrm{TM}}$ Reductant buffer and $10 \mu \mathrm{l}$ FOCUS ${ }^{\mathrm{TM}}$ Protein Reductant (or $250 \mathrm{mM}$ TCEP in DDI water) to $487.5 \mu \mathrm{l}$ DDI water.

b. Add $50 \mu \mathrm{l}$ reduction solution to tubes $B$ and $C$, and then suspend the pellet by gently pipetting up and down. Here, the pellet is not completely dissolved.

c. Incubate the tubes at $55^{\circ} \mathrm{C}$ for $1 \mathrm{~h}$. Vortex for $10 \mathrm{~s}$ every $10 \mathrm{~min}$ and confirm the complete dissolution of pellet after incubation. 
6. mBB labeling

a. Add $25 \mu \mathrm{l}$ of $60 \mathrm{mM} \mathrm{mBB}$ solution to tubes $\mathrm{A}, \mathrm{B}$, and $\mathrm{C}$.

Note: TCEP can be involved in dehalogenation of $M B B$ and might weaken the efficiency of fluorescent labeling in solution (Graham et al., 2003). Be sure to keep the mBB concentration higher than that of TCEP in the labeling solution.

b. Incubate at room temperature (approximately $23^{\circ} \mathrm{C}$ ) for $15 \mathrm{~min}$.

c. To remove excess unreacted $\mathrm{mBB}$, add an equal volume of cold $20 \%(\mathrm{w} / \mathrm{v}) \mathrm{TCA}$ solution to tubes $\mathrm{A}, \mathrm{B}$, and $\mathrm{C}$.

d. Centrifuge the tubes at $14,000 \times g, 4{ }^{\circ} \mathrm{C}$ for $30 \mathrm{~min}$.

e. Remove the supernatant, leaving the protein pellet intact.

f. Remove TCA by washing pellet twice each with $500 \mu \mathrm{l}$ cold acetone and retain the pellet.

7. Separation of the labeled proteins

a. Suspend the pellets in 10-25 $\mu \mathrm{l}$ SDS-PAGE sample buffer (can be stored at room temperature for 1 week, Recipe 3 ) and boil for $3 \mathrm{~min}$.

b. Assemble the Mini-PROTEAN TGX gel in Mini-PROTEAN Tetra system and pour SDSPAGE buffer (can be stored at room temperature for 1 month, Recipe 4).

c. Load the boiled samples, equivalent to $20-40 \mu \mathrm{g}$ of proteins, to the sample well.

Note: The initial amount of protein per tube is approximately $50 \mu \mathrm{g}$. Adjust and determine the loading volume based on some pilot experiments in the laboratory. We recommend setting an experimental replicate for SDS-PAGE with approximately $20 \mu \mathrm{g}$ protein, for example, a replicate with $10 \mu \mathrm{l}$ of the solution obtained by resuspending the pellet in $25 \mu \mathrm{l}$ SDS-PAGE sample buffer.

d. Start the electrophoretic run at $100 \mathrm{~V}$ and stop after the samples have traversed the desired distance.

8. Protein visualization

a. Place the gel on UV transilluminator and irradiate the gel.

b. Use yellow filter to obtain fluorescence derived from mBB-labeled protein.

Note: $\mathrm{mBB}$ has an excitation maximum at $394 \mathrm{~nm}$ and an emission maximum at $490 \mathrm{~nm}$.

\section{Data analysis}

1. Interpretation of the difference between wild type (Col-0) and mutant is based on densitometric analysis of bands with conventional monochrome photographs or fluorescence images using any conventional software, such as ImageJ (https://imagej.nih.gov/ij//).

2. The representative data indicate comparable band intensity in $\mathrm{R}-\mathrm{SH}$ staining between Col-O and the mutant (Figure 2). However, only on the basis of R-SH data, we cannot assess the redox state of thiol-containing proteins. Here, the R-S-S-R data show a slight increase in band intensity in the mutant and the "Total" data show a slight decrease in band intensity (Figure 2), suggesting that the mutant has an oxidizing atmosphere for thiol-containing proteins. The combined 
interpretation clearly raises the possibility that the mutant has an oxidizing atmosphere in thiolcontaining proteins and the redox state may contribute to the decrease in the amount of thiolcontaining proteins.

3. Figure 3 exemplifies several different cases to be assumed. Case $A$ indicates comparable band intensities in R-SH staining between Col-0 and the mutant. Case B indicates the R-SH data, showing a decrease in band intensity in the mutant and Case $\mathrm{C}$ indicates the $\mathrm{R}$-SH data, showing an increase in band intensity in the mutant.

4. In all these cases, interpretations about the redox state can be changed by the R-S-S-R data and the "Total" data (compare cases $A 1$ to $A 2, B 1$ to $B 2$, and $C 1$ to $C 2$ ).

5. For example, in Case B1, the R-S-S-R data demonstrating an increase in band intensity in the mutant support the interpretation based on the R-SH data, suggesting an oxidizing atmosphere in the mutant. On the other hand, in Case B2, the R-S-S-H data demonstrating a decrease in band intensity in the mutant is contrary to the hypothesis based on the R-SH data. The total (R$\mathrm{SH}$ and $\mathrm{R}-\mathrm{S}-\mathrm{S}-\mathrm{R}$ ) data represent that the mutant has lesser variety of thiol-containing proteins. The combined interpretation raises the possibility that the mutant has a comparable redox state in thiol-containing proteins as Col-0 and, thereby, the free thiol might decrease in Case B2.

6. Many Arabidopsis mutants have a variety of proteome, resulting from comprehensive proteomic reorganization in response to mutations in various regulatory networks (Figure 3, Case B2 and C1). To minimize possible misinterpretations regarding the redox state of thiol-containing proteins, it is desirable to obtain not only the R-SH data, but also the R-S-S-R and total thiol data.

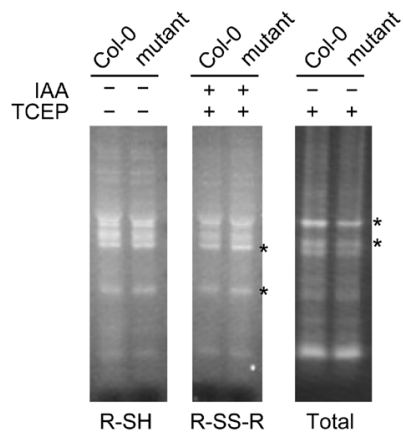

Figure 2. Representative data for monobromobimane (mBB) staining of thiol-containing proteins. Free thiols $(\mathrm{R}-\mathrm{SH})$ in proteins were detected by $\mathrm{mBB}$ staining without alkylation or reduction (IAA: -, TCEP: -). Disulfide bonds (R-S-S-R) were stained by $m B B$ followed by alkylation and reduction (IAA: +, TCEP: +). Total thiols (R-SH and R-S-S-R) were visualized with mBB only after reduction (IAA: -, TCEP: +). Asterisks indicate different band intensities in Col0 and the mutant. 

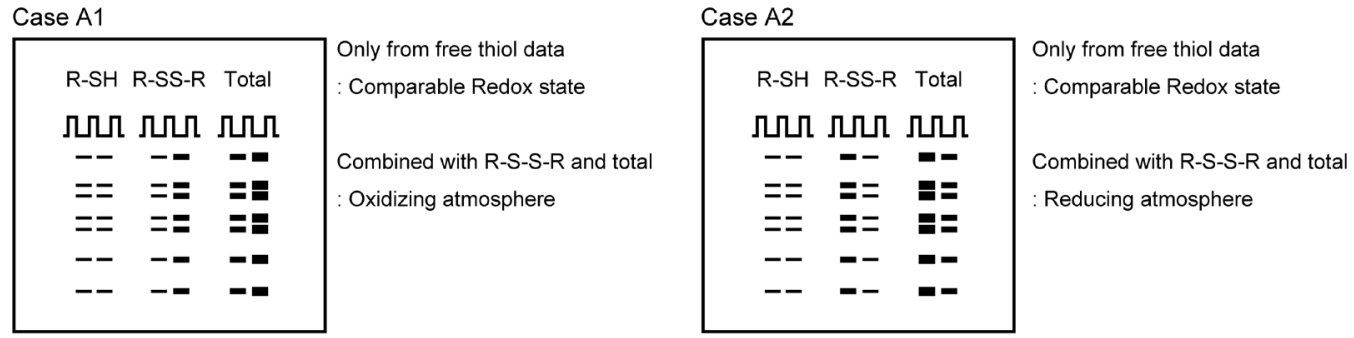

Case B1

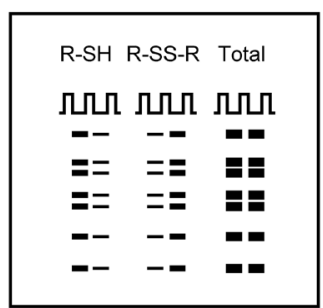

Only from free thiol data
: Oxidizing atmosphere
Combined with R-S-S-R and total
: Oxidizing atmosphere

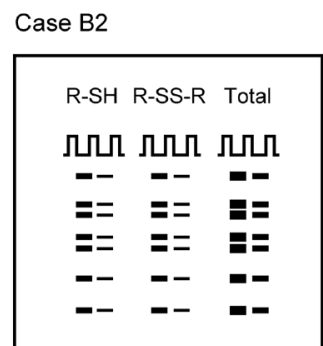

Only from free thiol data : Oxidizing atmosphere

Combined with R-S-S-R and total : Decrease of thiol-containing proteins

Case C1

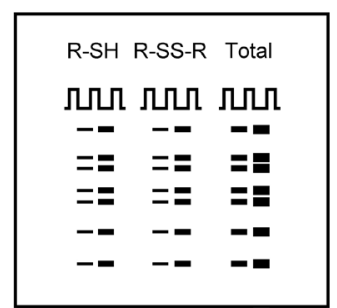

Only from R-SH Reducing atmosphere Combined with R-S-S-R and total Increase of thiol-containing proteins (a)
Case C2

\begin{tabular}{|c|c|c|}
\hline $\mathrm{R}-\mathrm{SH}$ & R-SS-R & Total \\
\hline 几ు & 几Ц & 几ు \\
\hline & & \\
\hline =E & $==$ & 트름 \\
\hline ニニ & Eニ & 틀 \\
\hline-- & $=-$ & $-=$ \\
\hline$-\boldsymbol{-}$ & $=-$ & $-=$ \\
\hline
\end{tabular}

Only from free thiol data : Reducing atmosphere Combined with R-S-S-R and total : Reducing atmosphere

Figure 3. Representative interpretation for the mutant of interest. Representative images of monobromobimane (mBB) stained gels. In all the cases, the left lane is of wild type (Col-0) and the right lane is of the mutant. R-SH and R-S-S-R indicate free thiols and disulfide bonds, respectively, in the proteins.

\section{Recipes}

1. Protein extraction buffer

$30 \mathrm{mM}$ Tris $/ \mathrm{HCl}, \mathrm{pH} 7.0$, with one tablet of $\mathrm{cOmplete}^{\mathrm{TM}}$, Mini Protease Inhibitor Cocktail per $10 \mathrm{ml}$

2. IAA solution

$$
0.4 \text { M IAA }
$$

3. SDS-PAGE sample buffer

$50 \mathrm{mM}$ Tris/HCl, $\mathrm{pH} 7.0$

$2 \%(\mathrm{w} / \mathrm{v}) \mathrm{SDS}$

$5 \%(\mathrm{w} / \mathrm{v})$ glycerol

$0.01 \%(w / v)$ BPB

4. SDS-PAGE buffer

\section{$250 \mathrm{mM}$ Tris}

\section{$1.92 \mathrm{M}$ glycine}

$1 \%(w / v)$ SDS 


\section{Acknowledgments}

This protocol was adapted and modified from previous work by Hashida et al. (2018). This work was supported by the JSPS KAKENHI Grant Number $17 \mathrm{H} 05714$ to M.K.Y.

\section{Competing interests}

The authors declare that there are no conflicts of interest.

\section{$\underline{\text { References }}$}

1. Fahey, R. C. and Newton, G. L. (1987). Determination of low-molecular-weight thiols using monobromobimane fluorescent labeling and high-performance liquid chromatography. Methods Enzymol 143: 85-96.

2. Fahey, R. C., Newton, G. L., Dorian, R. and Kosower, E. M. (1980). Analysis of biological thiols: derivatization with monobromotrimethylammoniobimane and characterization by electrophoresis and chromatography. Anal Biochem 107(1): 1-10.

3. Fahey, R. C., Newton, G. L., Dorian, R. and Kosower, E. M. (1981). Analysis of biological thiols: quantitative determination of thiols at the picomole level based upon derivatization with monobromobimanes and separation by cation-exchange chromatography. Anal Biochem 111(2): 357-365.

4. Fenton, S. S. and Fahey, R. C. (1986). Analysis of biological thiols: determination of thiol components of disulfides and thioesters. Anal Biochem 154(1): 34-42.

5. Getz, E. B., Xiao, M., Chakrabarty, T., Cooke, R. and Selvin, P. R. (1999). A comparison between the sulfhydryl reductants tris(2-carboxyethyl)phosphine and dithiothreitol for use in protein biochemistry. Anal Biochem 273(1): 73-80.

6. Graham, D. E., Harich, K. C. and White, R. H. (2003). Reductive dehalogenation of monobromobimane by tris(2-carboxyethyl)phosphine. Anal Biochem 318(2): 325-328.

7. Hashida, S. N., Miyagi, A., Nishiyama, M., Yoshida, K., Hisabori, T. and Kawai-Yamada, M. (2018). Ferredoxin/thioredoxin system plays an important role in the chloroplastic NADP status of Arabidopsis. Plant J 95(6): 947-960.

8. Meyer, A. J., May, M. J. and Fricker, M. (2001). Quantitative in vivo measurement of glutathione in Arabidopsis cells. Plant $J$ 27(1): 67-78.

9. Newton, G. L., Dorian, R. and Fahey, R. C. (1981). Analysis of biological thiols: derivatization with monobromobimane and separation by reverse-phase high-performance liquid chromatography. Anal Biochem 114(2): 383-387.

10. Riddles, P. W., Blakeley, R. L. and Zerner, B. (1983). Reassessment of Ellman's reagent. Methods Enzymol 91: 49-60. 
11. Tyagarajan, K., Pretzer, E. and Wiktorowicz, J. E. (2003). Thiol-reactive dyes for fluorescence labeling of proteomic samples. Electrophoresis 24(14): 2348-2358. 